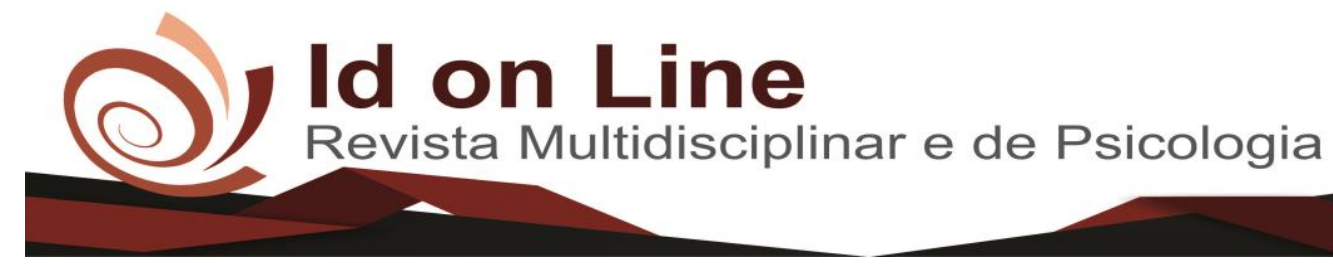

DOI: $10.14295 /$ idonline.v14i49.2332

Artigo

\title{
Nível de Compreensão de Pacientes Internados em um Hospital da Região Metropolitana do Cariri-CE sobre os Medicamentos Prescritos na Alta Hospitalar
}

\author{
Gabriella Gonçalves Feitosa ; José Leonardo Gomes Coelho ${ }^{2}$; Willma José de Santana ${ }^{3,4}$; Rafael de Carvalho \\ Mendes $^{3,5}$; Emanuela Machado Silva Saraiva ${ }^{3}$; Francisca Eritânia Passos Rangel ${ }^{3}$
}

\begin{abstract}
Resumo: Objetivando avaliar o nível de compreensão de pacientes hospitalizados em um Hospital da Região Metropolitana do Cariri-CE sobre os medicamentos prescritos na alta hospitalar, trata-se de um estudo exploratório, descritivo com abordagem quantitativo. Foram entrevistados 38 pacientes maiores de 18 anos que receberam alta hospitalar no mês de abril de 2019, por meio de um questionário aplicado de forma individual e reservada, em um Hospital localizado na Região do Cariri Cearense. Os resultados mostraram que $29 \%$ dos entrevistados não receberam orientações do médico no momento da alta hospitalar sobre o uso dos medicamentos em casa, $76 \%$ afirmaram que o médico não perguntou sobre alergia medicamentosa, $47 \%$ não sabem sobre efeitos adversos, $45 \%$ já interromperam ou trocaram a medicação por conta própria, mostrando que o nível de conhecimento dos pacientes sobre a utilização de medicamentos prescritos na alta hospitalar é ruim. Portanto, faz-se necessário ações de conscientização direcionadas aos prescritores enfatizando a necessidade de melhorar as informações passadas para o paciente no momento da alta hospitalar.
\end{abstract}

Palavras-chaves: Alta do paciente; Conhecimento. Cuidados Farmacêuticos; Tratamento Farmacológico.

\section{Understanding Level of Hospitalized Patients in a Hospital in the Metropolitan Region of Cariri-CE About the Prescribed Drugs at Hospital Discharge}

\begin{abstract}
Aiming to evaluate the level of understanding of patients hospitalized in a Hospital in the Metropolitan Region of Cariri-CE about the drugs prescribed at hospital discharge, this is an exploratory, descriptive study with a quantitative approach. 38 patients over 18 years old who were discharged in April 2019 were interviewed, through a questionnaire applied individually and privately, in a Hospital located in the Cariri Cearense Region. The results showed that $29 \%$ of respondents did not receive guidance from the doctor at the time of hospital discharge on the use of medicines at home, $76 \%$ stated that the doctor did not ask about drug allergy, $47 \%$ do not know about adverse effects, $45 \%$ have already stopped or switched medication on their own, showing that patients' level of knowledge about the use of prescription drugs at hospital discharge is poor. Therefore, it is necessary to raise awareness actions aimed at prescribers, emphasizing the need to improve the information given to the patient at the time of hospital discharge.
\end{abstract}

Keywords: Patient discharge; Knowledge. Pharmaceutical Care; Pharmacological treatment.

\footnotetext{
${ }^{1}$ Graduada em Farmácia pela Faculdade de Juazeiro do Norte - FJN. gabriellagf97@ hotmail.com;

${ }^{2}$ Discente em Farmácia da Faculdade de Juazeiro do Norte - FJN. leonardo-coelho-10@ hotmail.com;

${ }^{3}$ Docentes da Faculdade de Juazeiro do Norte -FJN. Docente da Faculdade de Tecnologia FATEC Cariri. wjsantana@hotmail.com;

${ }^{4}$ Docente da Faculdade de Tecnologia FATEC Cariri. ${ }^{5}$ Docente da Faculdade de Medicina do Juazeiro do Norte - FMJ. rafa_mendes@msn.com;

${ }^{5}$ Docentes da Faculdade de Juazeiro do Norte -FJN. Docente da Faculdade de Tecnologia FATEC Cariri. emanuelams@hotmail.com;

${ }^{6}$ Docente da Faculdade de Medicina do Juazeiro do Norte - FMJ. eritaniarangel@yahoo.com.br
} 


\section{Introdução}

A farmacoterapia dispõe um importante papel na prevenção, manutenção e recuperação da saúde, colaborando para aumentar a qualidade e perspectiva de vida da população. Mas, ainda existem muitas pessoas que não têm um conhecimento apropriado ou suficiente quanto ao uso correto de medicamentos, como, por exemplo, modo de administração e armazenamento, uso ou não de medicamentos com alimentos, clareza de uma prescrição médica e automedicação (LUPATINI et al., 2016).

Os medicamentos possuem eficácia, segurança e qualidade, e trazem importantes benefícios favorecendo a população. Além disso, o medicamento é uma forma prevenir e curar doenças resultando em bem-estar, proporcionando uma melhoria da saúde e da qualidade de vida dos indivíduos (LACERDA; MONTEIRO, 2016).

A falta de conhecimento dos pacientes relacionados com os medicamentos pode refletir a comunicação entre médico e paciente. As informações ao paciente devem ser fornecidas de forma clara e preferentemente por escrito, pois a não-compreensão da prescrição pode resultar em erros de medicação e riscos à saúde do paciente. Aconselha-se que o paciente receba informações sobre a identidade do medicamento, a indicação terapêutica, a administração, a durabilidade do tratamento, efeitos adversos e precauções, entre outros (FROHLICH et al., 2010).

Em momentos de transição do cuidado, como a alta hospitalar, os pacientes podem estar particularmente vulneráveis a ocorrência de eventos adversos relativos a medicamentos. Estudos demonstram que ações como orientação ao paciente e/ou cuidador e seguimento domiciliar são desenvolvidas para reduzir os riscos de ocorrência de eventos adversos relacionados ao uso de medicamentos após a alta hospitalar, cooperando para impedir danos à saúde dos pacientes (MARQUES et al., 2017).

O paciente bem informado decide junto com o médico sobre como tratar uma doença, sendo uma mudança radical na estrutura da relação entre profissional e paciente. Entretanto isso depende da orientação adequada feita por enfermeiros, médicos e farmacêuticos, podendo ajudar na prevenção de outros eventos adversos relacionados aos medicamentos (CASTRO; MELO, 2015).

Segundo Aquino et al. (2017), adesão à terapia medicamentosa pode ser compreendida como a extensão com a qual o comportamento do indivíduo - uso dos medicamentos, 
seguimento de uma dieta, e/ou execução de mudanças no estilo de vida coincidem com as recomendações dos profissionais de saúde.

A ocorrência de problemas relacionados a medicamentos é particularmente comum após alta hospitalar, quando múltiplas alterações no tratamento medicamentoso do paciente podem ser acompanhadas por inadequação na orientação e seguimento do paciente. Esses fatores comumente resultam na prescrição inapropriada de medicamentos, discrepâncias entre o regime medicamentoso prescrito após alta hospitalar e o tratamento anterior à internação, problemas de adesão ao tratamento, e inadequada vigilância de efeitos adversos (FURTADO; MARQUES; MONACO, 2010).

A presente pesquisa justifica-se a falta de informação do paciente com relação ao seu tratamento com medicamentos com o início de ação do medicamento, tempo de tratamento ou a não compreensão das informações recebidas dos profissionais da saúde podem ser determinantes para a não-adesão involuntária do paciente ao tratamento. Portanto, o cuidado Farmacêutico no âmbito hospitalar beneficia cada vez mais a segurança do paciente, ao contribuir para aumentar o conhecimento do paciente a respeito dos medicamentos prescritos na alta hospitalar, garantindo analisar prescrições, avaliando as prováveis interações, assegurando aprovação ao tratamento e contribuindo para a o uso racional dos medicamentos.

O presente estudo tem como objetivo avaliar o nível de compreensão de pacientes hospitalizados em um Hospital da Região Metropolitana do Cariri-CE sobre os medicamentos prescritos na alta hospitalar, bem como conhecer o perfil dos pacientes que recebem alta hospitalar (idade, sexo, nível de escolaridade, renda, estado cívil, residência); Identificar os principais medicamentos prescritos na alta hospitalar; Identificar se os pacientes receberam orientação farmacêutica no momento da alta hospitalar; Verificar o nível de conhecimento dos pacientes sobre a indicação, dose, frequência de administração, duração do tratamento e possíveis efeitos adversos dos medicamentos prescritos na alta hospitalar.

\section{Metodologia}

Tratou-se de um estudo do tipo descritivo, exploratório e quantitativo. O estudo foi realizado em um hospital privado na Região do Cariri Cearense. A amostra do estudo foi composta por 38 pacientes que receberam alta hospitalar no mês de abril de 2019 . 
Foram incluídos no estudo pacientes maiores de 18 anos, de ambos os sexos, que ficaram internados por no mínimo 24 horas e que ao receber alta hospitalar, apresentaram prescrição médica contendo no mínimo um medicamento para uso domiciliar. Foram excluídos pacientes com alta hospitalar sem prescrição de medicamentos para uso domiciliar ou que estiveram hospitalizados somente em observação.

Os dados foram coletados no mês de abril de 2019 através de questionários respondidos pelos pacientes mediante orientações, e foram transferidos posteriormente para uma ficha padronizada elaborada pelo próprio pesquisador para tal finalidade.

A partir das informações colhidas foi construído um banco de dados contendo as variáveis propostas pela pesquisa onde os dados foram analisados através do Microsoft Excel 2010 para consolidação dos resultados.

Os riscos de constrangimento em responder as perguntas foram minimizados direcionando os pacientes para uma sala reservada para fazer a entrevista. O anonimato de cada paciente foi garantido, sendo preservada suas informações pessoais. Com benefício de contribuir para melhorar o conhecimento dos pacientes com alta hospitalar sobre a importância de conhecer como utilizar corretamente os medicamentos prescritos para uso domiciliar que impactam diretamente no processo de adesão da farmacoterapia.

O presente estudo foi desenvolvido mediante aprovação do Comitê de Ética e Pesquisa da Faculdade de Juazeiro do Norte - FJN sob parecer consubstanciado de n³.215.148, atendendo as orientações sugeridas na Resolução № 466, de 12 de dezembro de 2012 do Conselho Nacional de Saúde.

\section{Resultados e Discussão}

Os resultados a seguir apresentam os achados encontrados mediante aplicação de questionários com 38 pacientes que receberam alta hospitalar em um Hospital da Região Metropolitana do Cariri-CE após internação e que continham prescrição de pelo menos um medicamento para uso domiciliar. 
Tabela 1 - Perfil sociodemográfico de pacientes pós-alta hospitalar -Região do Cariri, abril 2019.

\begin{tabular}{|c|c|c|}
\hline VARIÁVEIS & $\mathbf{N}$ & $\%$ \\
\hline \multicolumn{3}{|l|}{ IDADE } \\
\hline 18 a 35 anos & 12 & $31,6 \%$ \\
\hline 36 a 55 anos & 11 & $28,5 \%$ \\
\hline 56 a 70 anos & 15 & $39,9 \%$ \\
\hline Total & 38 & $100,0 \%$ \\
\hline \multicolumn{3}{|l|}{ SEXO } \\
\hline Feminino & 20 & $52,6 \%$ \\
\hline Masculino & 18 & $47,4 \%$ \\
\hline Total & 38 & $100 \%$ \\
\hline \multicolumn{3}{|c|}{ NÍVEL DE ESCOLARIDADE } \\
\hline Fundamental completo & 7 & $18,5 \%$ \\
\hline Médio completo & 14 & $36,9 \%$ \\
\hline Superior completo & 4 & $10,5 \%$ \\
\hline Outros & 13 & $34,2 \%$ \\
\hline Total & 38 & $100 \%$ \\
\hline \multicolumn{3}{|l|}{ RENDA } \\
\hline Até 1 salário mínimo & 11 & $28,9 \%$ \\
\hline De 1 a 3 salários mínimos & 20 & $52,6 \%$ \\
\hline 4 salários mínimos ou mais & 5 & $13,2 \%$ \\
\hline Sem renda fixa & 2 & $5,3 \%$ \\
\hline Total & 38 & $100 \%$ \\
\hline \multicolumn{3}{|l|}{ ESTADO CIVIL } \\
\hline Solteiro & 8 & $21,1 \%$ \\
\hline Casado & 23 & $60,5 \%$ \\
\hline Viúvo & 1 & $2,6 \%$ \\
\hline Outros & 6 & $15,8 \%$ \\
\hline Total & 38 & $100 \%$ \\
\hline \multicolumn{3}{|l|}{ RESIDÊNCIA } \\
\hline Zona urbana & 31 & $81,6 \%$ \\
\hline Zona rural & 7 & $18,4 \%$ \\
\hline Total & 38 & $100 \%$ \\
\hline
\end{tabular}

Fonte: próprio autor.

Conforme a Tabela 1, verificou-se que 20 dos entrevistados $(\mathrm{p}=52,6 \%)$ eram do sexo feminino e $18(\mathrm{p}=52,6 \%)$ do sexo masculino, a faixa de idade foi de 18 a 70 anos. Em relação a escolaridade $7(\mathrm{p}=18,5 \%)$ apresentavam ensino fundamental completo; $14(\mathrm{p}=36,9 \%)$ médio completo; $4(\mathrm{p}=10,5 \%)$ superior completo, $13(\mathrm{p}=34,2 \%)$ outros. A maior parte estava empregada no momento da entrevista e a maior renda foi evidenciada com 20 entrevistados 
( $\mathrm{p}=52,6 \%$ ) de 1 a 3 salários mínimos. Em relação ao estado civil, 23 ( $\mathrm{p}=60,5 \%)$ eram casados, $8(\mathrm{p}=21,1 \%)$ solteiros, $1(2,6 \%)$ viúvos e $6(15,8 \%)$ outros.

Tabela 2 - Motivos que levaram a internação. Região do Cariri, abril 2019.

\begin{tabular}{lcc} 
MOTIVO DA INTERNAÇÃO & N & \% \\
Gastroenterites & 9 & $23,7 \%$ \\
Hipertensão & 6 & $15,8 \%$ \\
Virose & 5 & $13,2 \%$ \\
Doenças respiratórias & 4 & $10,5 \%$ \\
Diabetes & 4 & $10,5 \%$ \\
Hipertensão + Diabetes & 3 & $7,9 \%$ \\
Outras & 7 & $18,4 \%$ \\
Total & 38 & $100,0 \%$ \\
\hline
\end{tabular}

Fonte: próprio autor.

Conforme a Tabela 2, das 38 pessoas entrevistadas após a alta hospitalar, 9 pacientes $(\mathrm{p}=23,7 \%)$ relataram o motivo da internação por gastroenterites, 6 pacientes $(\mathrm{p}=15,8 \%)$ por problemas de pressão arterial sistêmica elevada, 5 pessoas $(\mathrm{p}=13,2 \%)$ motivadas por virose, 4 pacientes $(\mathrm{p}=10,5 \%)$ por diabetes, $4(\mathrm{p}=10,5)$ com problemas respiratórios, $3(\mathrm{p}=7,9 \%)$ pacientes com Hipertensão + Diabetes descompensadas e 7 pacientes $(\mathrm{p}=18,4 \%)$ por outros problemas de saúde.

Os dados encontrados na presente pesquisa corroboram com o estudo realizado por Aquino et al., (2010), que tinha como objetivo analisar as tendências das principais causas de internações hospitalares entre aquelas sensíveis à atenção primária, onde os dados secundários foram provenientes do Sistema de Informação Hospitalar (SIH-SUS) e do senso demográfico do ano de 2001 e projeções populacionais do Instituto Brasileiro de Geografia e Estatística (IBGE), no qual, foi observado que as gastroenterites foram a primeira causa de internação em todas as faixas etárias.

Em contrapartida, os achados desde estudo foram contrários aos encontrados em um estudo realizado por Santos \&Vasconselos (2013) durante os anos de 2008 a 2012, em que foram registradas 436.316 internações por hipertensão arterial em caráter de urgência e emergência, na faixa etária de 20 anos ou mais. 
Tabela 3 -Relação de doenças crônicas encontradas. Região do Cariri, abril 2019.

\begin{tabular}{lcc} 
DOENÇA CRÔNICA PREDOMINATE & N & $\%$ \\
Hipertensão & 6 & $15,80 \%$ \\
Hipertensão + Diabetes & 5 & $13,15 \%$ \\
Diabetes & 3 & $7,90 \%$ \\
Outras & 7 & $18,42 \%$ \\
Não possui doenças crônicas & 17 & $44,73 \%$ \\
Total & 38 & $100 \%$ \\
\hline
\end{tabular}

Fonte: próprio autor.

Conforme a Tabela 3, dos 38 entrevistados pós-alta hospitalar, observou-se que 21 pacientes $(\mathrm{p}=55,27 \%)$ relataram ter alguma doença crônica, enquanto 17 pacientes $(44,73 \%)$ não se enquadravam nesse quesito.

Quando perguntados sobre qual era doença crônica predominante, a maior frequência foi de hipertensão 6 pacientes $(\mathrm{p}=15,80 \%), 5$ pacientes $(\mathrm{p}=13,15 \%)$ hipertensão + diabetes, 3 pacientes $(\mathrm{p}=7,90 \%)$ diabetes e 7 pacientes $(\mathrm{p}=18,42 \%)$ outras comorbidades.

No estudo realizado por Oliveira (2010), no qual, foi selecionado usuários admitidos com diagnóstico de Hipertensão e/ou Diabetes, com 20 anos ou mais, de ambos os sexos, residentes ou não no município de Dourados-MS e feita uma amostragem por conveniência, de 200 pacientes entrevistados observou-se que $94,5 \%$ eram hipertensos.

Nesse contexto, é importante enfatizar que pacientes que recebem informações adequadas sobre o uso correto dos medicamentos de uso domiciliar, tendem a uma maior adesão na terapia medicamentosa. Assim, Barros (2014), enfatiza que em relação à hipertensão arterial sistêmica, alguns fatores podem estar relacionados com a adesão do paciente ao tratamento, ressaltando-se a falta de conhecimento sobre a doença, aos medicamentos e motivação para tratar uma doença crônica

Tabela 4 - Medicamentos prescritos ou de uso domiciliar. Região do Cariri, abril, 2019.

\begin{tabular}{llc}
\hline VARIÁVEIS & N & \% \\
MEDICAMENTOS PRESCRITOS PARA CASA & & $6,7 \%$ \\
Metformina & 6 & $5,6 \%$ \\
Hidroclorotiazida & 5 & $5,6 \%$ \\
Dipirona & 5 & $4,4 \%$ \\
Vitamina C & 4 & $4,4 \%$ \\
Ciprofloxacino & 4 & $4,4 \%$ \\
Repoflor & 4 & $3,3 \%$ \\
Captopril & 3 &
\end{tabular}




\begin{tabular}{|c|c|c|}
\hline Enalapril & 3 & $3,3 \%$ \\
\hline Paracetamol & 3 & $3,3 \%$ \\
\hline Furosemida & 3 & $3,3 \%$ \\
\hline Atenolol & 3 & $3,3 \%$ \\
\hline Pantoprazol & 3 & $3,3 \%$ \\
\hline Sinvastatina & 3 & $3,3 \%$ \\
\hline Glibenclamida & 3 & $3,3 \%$ \\
\hline Outros & 38 & $42,2 \%$ \\
\hline Total & 90 & $100 \%$ \\
\hline \multicolumn{3}{|c|}{ MEDICAMENTOS JÁ UTILIZADOS EM CASA } \\
\hline Metformina & 5 & $12,2 \%$ \\
\hline Hidroclorotiazida & 4 & $9,8 \%$ \\
\hline Enalapril & 3 & $7,3 \%$ \\
\hline Furosemida & 3 & $7,3 \%$ \\
\hline Losartana & 2 & $4,9 \%$ \\
\hline Atenolol & 2 & $4,9 \%$ \\
\hline Captopril & 2 & $4,9 \%$ \\
\hline Sinvastatina & 1 & $2,4 \%$ \\
\hline Glibenclamida & 1 & $2,4 \%$ \\
\hline Xarelto & 1 & $2,4 \%$ \\
\hline Outros & 17 & $41,5 \%$ \\
\hline Total & 41 & $100,0 \%$ \\
\hline \multicolumn{3}{|c|}{$\begin{array}{l}\text { NÚMERO DE MEDICAMENTOS POR } \\
\text { PRESCRIÇÃO PARA CASA }\end{array}$} \\
\hline 1 medicamento & 6 & $15,8 \%$ \\
\hline 2 medicamentos & 11 & $28,9 \%$ \\
\hline 3 medicamentos & 10 & $26,3 \%$ \\
\hline 4 medicamentos & 10 & $26,3 \%$ \\
\hline 5 ou mais medicamentos & 1 & $2,6 \%$ \\
\hline Total & 38 & $100,0 \%$ \\
\hline
\end{tabular}

Fonte: próprio autor.

Conforme a Tabela 4, observa-se que a maior parte dos medicamentos prescritos para utilização domiciliar, são medicamentos anti-hipertensivos, hipoglicemiantes e os antiinflamatórios não esteroides (AINES). Dentre estes os mais frequentes foram: metformina prescrito para 6 pacientes $(\mathrm{p}=6,7 \%)$; hidroclorotiazida, dipirona, foram prescritos para 5 pacientes $(\mathrm{p}=5,6 \%)$; vitamina $\mathrm{C}$, ciprofloxacino e repoflor para $4(\mathrm{p}=4,4 \%)$; enalapril, captopril, furosemida, pantoprazol, sinvastatina, atenolol, para $3(\mathrm{p}=3,3 \%)$.

De todos os medicamentos já utilizados em casa observa-se de uma forma geral que a maior parte é para tratamento de hipertensão e uma menor parte para tratamento do diabetes. Sendo os mais utilizados: Metformina por $5(\mathrm{p}=12,2 \%)$ dos pacientes (tratamento do diabetes), 
enquanto que hidroclorotiazida $4(\mathrm{p}=9,8 \%)$, enalapril e furosemida $3(\mathrm{p}=7,3 \%)$ pacientes (tratamento de hipertensão).

Das 38 pessoas entrevistadas, todas receberam prescrições de medicamentos no ato da alta hospitalar, destaca-se que para $11(\mathrm{p}=28,9 \%)$ dos pacientes foram prescritos 2 medicamentos para utilizar em casa, enquanto para 10 pacientes $(\mathrm{p}=26,3 \%)$ foram prescritos 3 medicamentos, e para outros 10 pacientes $(\mathrm{p}=26,3 \%)$ foi prescrito 4 medicamento para fazer uso em casa, para 6 paciente $(\mathrm{p}=15,8 \%)$ foi prescrito somente um medicamento e para 1 paciente $(\mathrm{p}=2,6 \%)$ foi prescrito 5 ou mais medicamentos para utilizar em casa.

Diante do exposto, embora os medicamentos contribuam para melhora da qualidade e aumento da expectativa de vida, de forma profilática ou de tratamento, seu uso pode gerar impactos negativos, como a ocorrência de interações medicamentosas (IM) indesejadas. Assim, quanto maior o número de medicamentos na terapêutica do indivíduo, maior a probabilidade da ocorrência de iatrogenia (BELEZI, et al., 2018).

Tabela 5 - Grau de conhecimento dos participantes sobre a utilização de medicamentos. Região do Cariri, abril, 2019.

\begin{tabular}{lcc}
\hline VARIÁVEIS & N & $\%$ \\
MEDICO EXPLICOU NA ALTA? & 27 & $71 \%$ \\
Sim & 11 & $29 \%$ \\
Não & 38 & $100 \%$ \\
Total & & \\
MEDICO PERGUNTOU SOBRE ALERGIA? & 9 & $24 \%$ \\
Sim & 29 & $76 \%$ \\
Não & 38 & $100 \%$ \\
Total & & \\
ORIENTAÇÃO DE OUTRO PROFISSIONAL? & 26 & $68 \%$ \\
Sim & 12 & $32 \%$ \\
Não & 38 & $100 \%$ \\
Total & & \\
QUAL PROFISSIONAL RESPONSÁVEL PELA & & $31 \%$ \\
ORIENTAÇÃO? & 8 & $35 \%$ \\
Farmacêutico & 9 & $35 \%$ \\
Enfermeiro & 9 & $100 \%$ \\
Técnico de enfermagem & 26 & \\
Total & & $29 \%$ \\
DÚVIDAS SOBRE USO DOS MEDICAMENTOS? & 11 & $71 \%$ \\
Sim & 27 & $100 \%$ \\
Não & 38 & \\
Total & & \\
\hline
\end{tabular}

Fonte: próprio autor. 
Conforme a Tabela 5, verificou-se que das 38 pessoas entrevistadas, $27(\mathrm{p}=71 \%)$ relataram que o médico prestou orientação durante a alta, enquanto uma menor parte 11 pacientes $(\mathrm{p}=29 \%)$ relatou que o médico não explicou sobre os medicamentos durante a alta.

Das 38 pessoas entrevistadas, apenas $9(\mathrm{p}=24 \%)$ relataram que o médico perguntou se possuía alergia a medicamentos. A maior parte dos entrevistados, 29 pacientes $(\mathrm{p}=76 \%)$ relatou que no momento da alta, não houve esse tipo de indagação por parte do prescritor.

Quando perguntado ao paciente se recebeu orientação de outro profissional sobre o uso dos medicamentos observa-se que 26 pacientes $(\mathrm{p}=68 \%)$ receberam no momento da alta enquanto 12 pacientes ( $\mathrm{p}=32 \%$ ) não recebeu orientação de outro profissional e dos 26 pacientes $9(p=35 \%)$ receberam orientação de um enfermeiro, $9(p=35 \%)$ receberam orientação de um técnico de enfermagem e $8(\mathrm{p}=31 \%)$ receberam orientação de um farmacêutico.

Quando perguntado sobre ter dúvidas de como usar medicamento em casa, a maior frequência, foi referente a ausência de dúvidas, totalizando 27 pacientes $(\mathrm{p}=71 \%)$, enquanto um percentual menor relatou ainda ter dúvidas quanto ao uso correto de medicamentos no âmbito domiciliar, totalizando 11 pacientes $(\mathrm{p}=29 \%)$.

No estudo realizado por Carvalho et al., (2016) com 217 pessoas, verificou-se que ( $\mathrm{p}=$ $75 \%$ ) dos entrevistados no ambulatório do hospital e $24,1 \%$ na faculdade de odontologia, relataram também não ter dúvidas quanto ao uso correto dos medicamentos prescritos incluindo o tempo de tratamento.

No contexto da alta hospitalar, a resolução no 585 do Conselho Federal de Farmácia regulamenta as atribuições clínicas do farmacêutico, dentre elas a alta farmacêutica, com o objetivo de promover o uso racional de medicamentos e otimizar a farmacoterapia, visando alcançar resultados definidos que melhorem a qualidade de vida do paciente. Assim, o farmacêutico clínico pode e deve atuar junto à equipe multiprofissional, prevenindo, detectando e resolvendo os problemas relacionados à terapia, tanto no período de internação, como na alta hospitalar. A atuação do farmacêutico na alta pode ocorrer de várias formas, como a conciliação medicamentosa; a identificação de problemas na adesão ao tratamento; a orientação nos diversos aspectos relacionados à terapia medicamentosa, contribuindo melhorar a adesão à terapia, reduzindo o surgimento de eventos adversos relacionados a medicamentos $\mathrm{e}$ diminuindo a necessidade de novas internações (ADRIANO, et al., 2016). 
Tabela 6 - Grau de conhecimentos dos participantes sobre a utilização de medicamentos após alta Hospitalar. Região do Cariri, abril, 2019.

\begin{tabular}{lcc}
\hline VARIÁVEIS & $\mathbf{N}$ & $\%$ \\
COMO INGERIR MEDICAMENTOS ORAIS? & & \\
Água & 26 & $68 \%$ \\
Chá & 4 & $11 \%$ \\
Leite & 7 & $18 \%$ \\
Vitaminas & 1 & $3 \%$ \\
Outros & 0 & $0 \%$ \\
Total & 38 & $100 \%$ \\
SABE SOBRE EFEITOS ADVERSOS? & & \\
Sim & 20 & $53 \%$ \\
Não & 18 & $47 \%$ \\
Total & 38 & $100 \%$ \\
TOMOU MEDICAMENTO POR CONTA PRÓPRIA? & & \\
Sim & 30 & $79 \%$ \\
Não & 8 & $21 \%$ \\
Total & 38 & $100 \%$ \\
MEDICAMENTOS PODEM CAUSAR PROBLEMAS? & & \\
Sim & 31 & $82 \%$ \\
Não & 7 & $18 \%$ \\
Total & 38 & $100 \%$ \\
TROCOU/PAROU MEDICAMENTOS POR CONTA? & & \\
Sim & 17 & $45 \%$ \\
Não & 21 & $55 \%$ \\
Total & 38 & $100 \%$ \\
SABE USAR OS DOIS PRIMEIROS MEDICAMENTOS? & & \\
Acertou os dois medicamentos & 27 & $71 \%$ \\
Acertou só um medicamento & 4 & $11 \%$ \\
Errou os dois medicamentos? & 38 & $18 \%$ \\
Total & & $100 \%$ \\
\hline Fonte prop & \\
\hline
\end{tabular}

Fonte: próprio autor.

De acordo com a Tabela 6 , dos 38 entrevistados, 26 pacientes $(p=68 \%)$, relataram ingerir medicamento com água, enquanto que os outros $(\mathrm{p}=32 \%)$ relataram ingerir medicamentos com outros líquidos como chás, leite e vitaminas. Do total, 20 pacientes $(\mathrm{p}=$ $53 \%$ ) relataram saber sobre o que são eventos adversos a medicamentos, enquanto 18 pacientes ( $\mathrm{p}=47 \%)$ não sabem sobre os eventos adversos.

No entanto, os eventos adversos relacionados a medicamentos podem levar a importantes agravos à saúde dos pacientes. Dessa maneira, orientar ao paciente durante a dispensação é muito importante para evitar, prevenir e minimizar os resultados provenientes de 
eventos adversos decorrentes das práticas de atenção em saúde associados ao cuidado (BEZERRA, et al., 2015).

Os dados da presente pesquisa são contrários ao estudo realizado por Blat; Oenning; Oliveira, et al (2011) na Unidade básica de Saúde do Munícipio de Grão Pará, em Santa Catarina, que ao entrevistar 111 pacientes, 70 (após consulta) e 41 (após dispensação), foi verificado que apenas $28,5 \%$ foram classificados com nível bom de conhecimento sobre os evento adversos.

Quando perguntado sobre o uso de medicamentos por conta própria, a maior parte dos entrevistados relatou que faz uso de medicamento por conta própria totalizando 30 pacientes ( $\mathrm{p}=79 \%$ ), enquanto um percentual menor relatou não fazer uso dessa prática totalizando 8 pacientes $(\mathrm{p}=\mathrm{n} 21 \%)$. Esse achado se assemelha ao do estudo realizado por Matos et al. (2018) com 270 indivíduos, sendo 81 adolescentes de um faixa etária de 15 a 70 anos, pode-se observar que $69,3 \%$ nos últimos 15 dias havia se automedicado.

Em contrapartida, os resultados desde estudo foram opostos aos encontrados em um estudo realizado por Domingues, et al., (2017), que tinha como objetivo estimar a prevalência e investigar fatores associados a automedicação em adultos no Distrito Federal, Brasil com 1.820 pessoas entrevistadas, 646 usaram pelo menos um medicamento; a prevalência da automedicação foi de $14,9 \%$.

Das 38 pessoas que foram entrevistadas 31 pacientes $(\mathrm{p}=82 \%)$ relataram que sabem que medicamentos podem causar problemas se tomados de maneira errada, enquanto 7 pacientes $(\mathrm{p}=18 \%)$ não sabem que medicamentos podem causar problemas.

Contudo, automedicação é entendida como uma prática de ingerir medicamentos sem aconselhamento e/ou acompanhamento de um profissional de saúde qualificado. Dessa maneira, a ingestão de medicamento pode trazer riscos à saúde quando utilizada de forma incorreta (GOULART; LAZARINI; SILVA, 2014).

Quando perguntado sobre parada ou troca de medicamentos por conta própria, a maior frequência dos pacientes relatou em parar ou trocar o medicamento por conta própria totalizando 21 pacientes ( $\mathrm{p}=55 \%$ ), enquanto um percentual menor relatou não cometer tal erro totalizando 17 pacientes $(\mathrm{p}=45 \%)$.

Parar ou trocar o medicamento por conta própria pode trazer prejuízo na adesão ao tratamento, especialmente pacientes com doenças crônicas o qual o dano pode ser bem maior, sendo capaz de agravar o problema de saúde do paciente por falta de informações como 
conhecer os riscos que o medicamento pode ocasionar quando interrompido o uso ou substituído por outro sem orientação de um profissional habilitado.

Quando perguntado se o paciente sabia como usar os dois primeiros medicamentos, um total de 27 pacientes ( $\mathrm{p}=71 \%$ ) acertou os dois medicamentos, enquanto 4 pacientes $(\mathrm{p}=11 \%)$, acertou somente como usar um medicamento e 7 pacientes $(\mathrm{p}=18 \%)$ errou os dois medicamentos relatando não saber como usar nenhum dos medicamentos.

Segundo Deuschle (2015), a orientação realizada por um Farmacêutico no momento da alta é de grande relevância, pois é nesse momento em que o paciente vai receber orientações sobre como usar o medicamento, a dose correta, o tempo de tratamento, riscos ou benefícios.

\section{Conclusão}

O estudo buscou avaliar o nível de compreensão de pacientes hospitalizados sobre os medicamentos prescritos na alta. Constatou-se que os pacientes possuíam faixa etária entre 18 a 70 anos, sendo mais predominantes pessoas maiores de 56 a 70 anos, totalizando um percentual de $39,9 \%$.

Observou-se que a gastroenterite foi a primeira causa de internação sendo $27,7 \%$ dos pacientes, enquanto Hipertensão Arterial Sistêmica elevada foi a segunda causa com um percentual de $15,8 \%$.

Em relação com o grau de conhecimentos dos pacientes sobre a utilização de medicamentos $29 \%$ afirmaram que o médico explicou durante a alta hospitalar, $76 \%$ o médico não perguntou se o paciente tinha alergia medicamentos, $29 \%$ dos pacientes têm dúvidas de como fazer uso em casa da medicação que foi passada, $47 \%$ dos entrevistados não sabem sobre efeito adverso, $79 \%$ fazem uso de medicamentos por conta própria, $18 \%$ responderam que medicamentos não causam problemas, $45 \%$ dos pacientes já trocaram ou interromperam a medicação por conta própria. Em relação ao uso correto dos dois primeiros medicamentos da prescrição, $18 \%$ dos pacientes não sabia como ser tomados nenhum dos medicamentos prescritos.

Algumas variáveis analisadas apresentaram resultados semelhantes com outros estudos feitos em vários lugares do Brasil. Como também colaboram para alicerçar outros trabalhos sobre nível de compreensão dos pacientes sobre os fármacos prescritos na alta hospitalar, assim como possibilitar a discussão sobre as ações que devem ser tomadas para realizar medidas 
preventivas sobre uso irracional de medicamentos que refletem exatamente na autoconfiança do paciente.

Portanto, é necessário treinamentos e palestras para os profissionais e pacientes para melhorar as informações ao paciente para esclarecer quanto ao uso dos medicamentos que foram prescritos para uso domiciliar.

\section{Referências}

ADRIANO, et al. Orientação farmacêutica na alta hospitalar de pacientes transplantados: estratégia para a segurança do paciente. Revista Einstein, v.14, n.3, p. 359-65, 2016.

AQUINO, et al. Fatores associados à adesão ao tratamento Farmacológico em idosos que utilizam medicamentos anti-hipertensivo. Revista Brasileira de Geriatria, Rio de Janeiro, v. 20 n. 1, p.116-127, 2017.

AQUINO, et al. Principais causas de internação por condições sensíveis à atenção primária no Brasil: uma análise por faixa etária e região. Revista Brasileira Saúde de Materno Infantil. Recife. p. 583-591, 2010.

BLATT, C. R; OENNING, D; OLIVEIRA, B, V. Conhecimento dos pacientes sobre os medicamentos prescritos após consulta médica e dispensação. Ciência \& Saúde Coletiva, v. 16, n. 17, p. 3277-3283, 2011.

BARROS, A. L. B; BEZERRA, A. S. M; LOPES, J. L. Adesão de pacientes hipertensos ao tratamento medicamentoso. Revista Brasileira de Enfermagem. v. 67, n. 4, p. 550, 2014.

BELEZI, et al. A terapêutica medicamentosa, interações potenciais e iatrogenia como fatores relacionados à fragilidade em idosos. Revista Brasileira de Geriatria e Gerontologia, v. 21 n.5, p. 610-619, Rio de Janeiro, 2018.

BEZERRA, et al. Conhecimento dos profissionais de saúde sobre eventos adversos em unidade de terapia intensiva. Revista de Enfermagem. v. 23, n. 4, p. 46 1-7. Rio de Janeiro, 2015.

CARVALHO, T.P., et al. Conhecimento dos pacientes sobre a prescrição medicamentosa no serviço de emergência. Revista Brasileira de Enfermagem. v.70, n.2, p.351-7, 2018.

CASTRO, L. L. C; MELO, D. O. A contribuição do farmacêutico para a promoção do acesso e uso racional de medicamentos essenciais no SUS. Revista Ciências e Saúde, 2015.

DEUSCHLE, et al. Importância da assistência e atenção farmacêutica non ambiente Hospitalar. Revista Ciência e Tecnologia, Rio Grande do Sul, v. 1, n.1, p. 20-28, 2015.

DOMINGUES, P. H. F., et al. Prevalência e fatores associados à automedicação em adultos no Distrito Federal: estudo transversal de base populacional. Revista Epidemiologia e Serviços de Saúde. v. 26, n.2, p. 319-330, 2017. 
FROHLICH, et al. Instrumento para avaliação do nível de conhecimento da prescrição na atenção primária. Revista Saúde Pública, 2010.

GOURLAR, F. C; LAZARINI, C. A; SILVA, F. M. Caracterização da prática de automedicação e fatores associados entre universitários do Curso de enfermagem. Revista Eletrônica de Enfermagem. v. 16, jul/set, 2014.

LACERDA, J. T; MONTEIRO, E. R. Promoção do uso racional de medicamentos uma proposta de modelo avaliativo da gestão Municipal. Saúde debate, v. 40, p. 101-116, out- dez 2016.

LATORRE, M. R. D. O. Tendência de hospitalizações por diabetes mellitus: implicações para o cuidado em saúde. Revista Acta paul Enfermagem. v.28, n. 95, p. 401-7. 2015.

LIEBER, N. S. R; MARQUES, L. F. G. Segurança do paciente no uso de medicamentos após a alta hospitalar: estudo explorativo. Saúde Soc. V. 23, n. 4, p. 1431-1444, São Paulo, 2014.

LUPATINI, E. O; MUNCK, A. K; VIEIRA, R. C. Percepções dos pacientes de um Hospital de ensino quanto à farmacoterapia e à orientação farmacêutica na alta. Revista Brasileira de Farmácia Hospitalar e Serviços de Saúde. São Paulo, v. 5, n. 3, 28-33, jul-set. 2014.

MARQUEZS, et al. Transição do cuidado do Hospital para o domicílio: Revisão Integrativa. Rev. Cogitare Enfermagem, v. 22, 2017.

MATOS, et al. Prevalência, perfil e fatores associados à automedicação em adolescentes e servidores de uma escola pública profissionalizante. Caderno Saúde Coletiva. v.26, n.1, p. 7883, Rio de Janeiro, 2018.

OLIVEIRA, S. G. Caracterização dos pacientes hipertensos e diabéticos em serviço de urgência e emergência no município de Dourados-MS. Escola Nacional de Saúde Pública, Campo Grande, setembro, 2010.

SANTOS, S. S; VASCONCELOS, D. F. S. Hospitalizações por hipertensão arterial essencial em caráter de urgência no Brasil, 2008- 2012. Revista Ciências Médica Biologia, Salvador, v.12, p. 465-471, dez. 2013.

SILVA, A. E. B. C., et al. Eventos Adversos a medicamentos em um hospital sentinela do Estado de Goiás, Brasil. Revista Latino Americana de Enfermagem, São Paulo, v. 19, n.2, p.1-9, 2011.

\section{Como citar este artigo (Formato ABNT):}

MONTANHA FILHO, José Pinheiro; SOARES, José Mauro Madeiros Velôso; SOUZA NETO; Moisés Ozório de; QUIRINHO, Márcio César Oliveira; SOUZA, Arlindo Nonato Morais de. Endividamento e Desempenho Acadêmico sob a perspectiva dos Discentes de Contabilidade. Id on Line Rev.Mult. Psic., Fevereiro/2020, vol.14, n.49, p. 412-426. ISSN: 1981-1179.

Recebido: 29/01/2019;

Aceito: 08/02/220. 